\title{
Elin Fredsted: Analyser af dansk og tysk talesprog. Oslo : Novus forlag, 1998
}

Das dänischsprachige Buch, dessen Titel sich ins Deutsche in etwa so übersetzen läßt: „Analysen von gesprochener Sprache im Dänischen und Deutschen”, bezweckt, die externe Kommunikation in der Tourismusbranche zu untersuchen, wobei der Begriff sprachliche Höflichkeit im Mittelpunkt steht. Die Arbeit basiert auf dem von der EU unterstützten, in den Jahren 1992-94 am Institut für Grenzforschung durchgeführten Projekt „Dänisch und Deutsch als Fremdsprachen im Handel und Gewerbe in Schleswig-Holstein und Nordschleswig“ und auf den langjährigen Erfahrungen der Vf.in als „Grenzgängerin“ zwischen der dänischen und der deutschen Sprache und Kultur. Vorausgeschickt sei die Verwunderung, daß diese gekürzte Fassung der (gleichnamigen?) Dissertation von 1996 nicht gleich auf deutsch erscheint. Es ist zwar auf der einen Seite zu begrüßen, daß eine an einer dänischen Universität eingereichte Abhandlung auf dänisch abgefaßt und das Dänische als Wissenschaftssprache so in Ehren gehalten wurde, man muß jedoch auf der anderen Seite die Frage stellen, was bei der Veröffentlichung der (bearbeiteten) Dissertation schwerer wiegt: die Behauptung der Rolle des Dänischen als Wissenschaftssprache oder aber die Ansprache eines internationalen Leserkreises, dem jetzt ein wichtiger Beitrag zur Diskursanalyse vorenthalten bleibt?

Im ersten Kapitel wird das zu untersuchende sprachliche Material vorgestellt. Es geht um 1) sieben Tonbandaufnahmen von touristischen Führungen u.a. in Lübeck und Glücksburg, in Tondern und auf der Insel Römö, 2) 84 transkribierte Informationsgespräche zwischen Touristen und Mitarbeitern in Fremdenverkehrsämtern, 3) Videoaufnahmen aus den Fremdenverkehrsämtern in Tondern und Friedrichstadt. So gibt es in dem umfassenden Material sowohl monologische als auch dialogische Texte.

Die Vf.in versucht eingangs, das Material zur „Fachsprache des Tourismus“ und zu „funktionalen Beschreibungsmodellen“ in Beziehung zu setzen. Was die Fachsprache betrifft, verweist sie auf das Modell von Lothar Hoffmann, das sie nicht unproblematisch findet. Bei den funktionalen Modellen referiert sie kurz die Theorien von Bühler, Jakobson und Möhn/Pelka.

Im zweiten Kapitel stellt Fredsted methodische Überlegungen an, wobei sie u.a. zu Begriffen wie Äußerung und Diskurs Stellung nimmt. Ausführlich referiert werden die pragmatischen Ansätze von Grice und Leech, wobei der Begriff der Höflichkeit im Vordergrund steht.

Fredsted stellt verschiedene ,optionality scales“ und ,indirectness scales” von Leech vor, die den Versuch darstellen, die graduellen Unterschiede von jeweils Direktheit und Höflichkeit skalar abzubilden. Zum Beispiel ist Fahr 
mich nach Hause. nach solchen Skalen direkter und weniger höflich als Wärst $\mathrm{Du}$ vielleicht so nett, mich nach Hause zu fahren. (S. 43). Die Vf.in überlegt, inwieweit solche Skalen angebracht sind, um die sprachliche Wirklichkeit zu beschreiben, wobei sie sich teilweise davon distanziert (S. 44).

Auch das Buch Politeness ${ }^{1}$ behandelt Fredsted eingehend. Zentral ist dabei die Vorstellung von den sogenannten FTA (face-threatening acts), die nach Brown/Levinson für eine Theorie der Höflichkeit von grundlegender Bedeutung sind. Im Gegensatz zu Brown/Levinson möchte Fredsted Höflichkeit nicht ausschließlich auf Sprachhandlungen begrenzen, bei denen es um „face“ geht. Höflichkeit sei, argumentiert die Vf.in zu Recht, eine Komponente, die es potentiell bei allen Sprachhandlungen geben kann.

Da der Kontext für die Beschreibung konkreter Texte eine wichtige Rolle spielt, wird er auch in die Überlegungen von Fredsted mit einbezogen. Die Vf.in stellt fest, daß nur wenige Äußerungen per se höflich oder unhöflich seien, entscheidend seien vielmehr die Äußerung und der Kontext (S. 58).

Im dritten Kapitel wird die Höflichkeit in Monologen anhand des umfangreichen Materials analysiert. Fredsted stellt fest, daß ,konventionelle Höflichkeitsindikatoren“ (S. 146) bei einer deutschen touristischen Führung eine größere Rolle spielen als bei einer vergleichbaren dänischen Führung.

Kapitel 4 befaßt sich mit Gesprächen in dänischen und deutschen Fremdenverkehrsämtern. Fredsted benutzt als Methode u.a. die Konversationsanalyse. Es wird erklärt, welche Erwartungen die Touristen und die Mitarbeiter an die Situation haben. Es gibt dabei mehrere sprachliche Unterschiede im Vergleich zum Alltagsgespräch.

Fredsted führt an, daß sie sich vom gesammelten Datenmaterial und nicht von Regeln oder Theorien habe leiten lassen (S. 154). Analysiert werden u.a. Gesprächseröffnungen, für die eine Typologie aufgestellt wird. Untersucht wird außerdem, wie Bitten und Fragen formuliert werden. Weniger Raum widmet die Vf.in der Frage, wie Gespräche beendet werden.

Am Ende des vierten Hauptkapitels werden die Ergebnisse zusammengefaßt. Die Gespräche in Fremdenverkehrsämtern können idealtypisch in drei Phasen eingeteilt werden, und zwar - was nicht erstaunt - in eine Initialphase, eine Medialphase und eine Finalphase. Es bestehen interkulturelle Unterschiede: Die Initialphase und die Finalphase sind im deutschen Zusammenhang weniger themenorientiert. Dafür sind sie aber phatischer und eher ritualisiert als die entsprechenden dänischen Teiltexte.

1 Brown, Penelope / Stephen C. Levinson (1987). Politeness - Some universals in language usage (= Studies in Interactional Sociolinguistics 4). Cambridge: Cambridge University Press. 
Die Ergebnisse des vierten Kapitels veranlassen die Vf.in dazu, die sechs Sprachfunktionen von Roman Jakobsen um eine zu erweitern, und zwar um die sogenannte „responsorientierte Sprachfunktion“ (S. 218). Als Begründung dafür führt die Vf.in ins Feld, daß es rein logisch betrachtet eine unendliche Anzahl von Sprachfunktionen gebe. Ferner stützt sich Fredsted auf Levinson, nach dem es bestimmte Redebeiträge gibt, bei denen Respons bezweckt wird.

Die Analyse von den Videoaufnahmen erfolgt im fünften Kapitel. Untersucht werden dabei u.a. Gesprächseröffnungen, verbale, parasprachliche und nonverbale Kommunikation. Fredsted gelangt zu dem Ergebnis, daß sich bei den Videoaufnahmen ,ein relatives Gleichgewicht zwischen den dänischen und den deutschen Sprechsituationen einstellt, sobald parasprachliche und nonverbale Aspekte der Kommunikation mit einbezogen werden“ (S. 345). Das ist so zu verstehen, daß das in den Kapiteln 3 und 4 festgestellte „Defizit“ an konventioneller Höflichkeit in dänischen Texten ,,in einem gewissen Maße von non-verbalen und parasprachlichen Markierungen von Höflichkeit kompensiert wird" (ebd.).

Das sechste Kapitel widmet Fredsted Krisen in der Kommunikation, und sie faßt in Kapitel 7 die Ergebnisse zusammen.

Fredsted geht in Analyser af dansk og tysk talesprog sehr gründlich vor, und zwar gilt dies sowohl im theoretischen Teil wie auch bei den Analysen. Die Ergebnisse sind durchaus interessant, wenn es darum geht, die externe Kommunikation in der Tourismusbranche zu untersuchen. Die von Fredsted eruierten Hauptunterschiede zwischen den beiden Sprachen scheinen plausibel.

Vor dem Hintergrund des komplexen Untersuchungsgegenstandes überrascht es jedoch nicht, daß man in einigen Punkten eine andere Auffassung vertreten könnte als Fredsted. Eine Stärke dieser Arbeit ist eben die, daß sie in hohem Maße zu weiteren Diskussionen anregt.

Problematisch sind u.E. die im Anschluß an Leech aufgestellten Höflichkeitsskalen, deren Aussagekraft denn auch von Fredsted teilweise in Frage gestellt wird. Die Untersuchungen Fredsteds scheinen eigentlich zu dem Ergebnis zu führen, daß es sich kaum lohnt, mit solchen Skalen zu arbeiten. Die Gründe dafür liefert Fredsted im siebten Kapitel, wo sie zu Recht feststellt, daß es nicht möglich sei, Höflichkeit per se zu isolieren, und daß Kontext, Kotext, Intonation und andere Faktoren eine entscheidende Rolle spielen.

Über die Pragmatik schreibt die Vf.in: „In der Pragmatik gibt es eine fast unbegrenzte Anzahl von Variablen und kombinatorischen Mustern. Das macht es unmöglich, eine überschaubare Menge von Regeln aufzustellen“ (unsere Übersetzung) (S. 336). Diese Auffassung teilt Fredsted mit vielen anderen Linguisten. Es ist ohne Zweifel richtig, daß es in der Morphologie und in der Syn- 
tax eine weitaus geringere Anzahl von Variablen gibt. Daraus läßt sich aber nicht schließen, daß es unmöglich ist, Regeln $\mathrm{zu}$ formulieren und diese $\mathrm{zu}$ systematisieren. Auch wenn es unmöglich sein sollte, Regeln zu formulieren was nicht auszuschließen ist -, besagt das noch lange nicht, daß es keine gibt. Auch die Pragmatik unterliegt einer Systematik, ohne die nichts gehen würde. $\mathrm{Ob}$ - oder besser: wann wir sie aber erkennen, ist eine andere, ja eigentlich die Frage. Wir schließen uns an dieser Stelle der Auffassung von Heinemann und Viehweger an:

Aus diesen situativen Rahmenbedingungen lassen sich nach unserer Auffassung relevante Situationstypen ableiten. Wir teilen nicht die Annahme vieler Linguisten, daß man es in der sprachlichen Kommunikation mit einer ,unübersehbaren Vielfalt unterschiedlicher Situationen" zu tun hat, daß folglich eine Typisierung des Situationswissens von vornherein ausgeschlossen ist. Wir vertreten im Gegensatz dazu - mit Schwarz $(1985,55)$ - die Meinung, daß ,jede aktuell in ihrer Konstellation einmalige Situation auf dem Hintergrund einer beschränkten Anzahl von funktionalen Situationstypen/-mustern erlebt und bewältigt wird, deren Grundzüge als kollektiv-/gruppenspezifisch gemeinsam gewußt vorausgesetzt werden können...“" $(1991,154){ }^{2}$

Der Titel von Fredsteds Buch Analyser af dansk og tysk talesprog ist u.E. etwas unglücklich. Erstens läßt der Titel kaum darauf schließen, daß es um das „Verhältnis zwischen linguistischer Strategie und pragmatischer Strategie“ (S. 326) geht, im besonderen um sprachliche Höflichkeit. Zweitens wird ja nur ein kleiner Teil der gesamten gesprochenen Sprache untersucht, nämlich die externe Kommunikation der Tourismusbranche, und sie weist ja - wie die Vf.in überzeugend darstellt - beträchtliche Unterschiede im Vergleich zu anderen Formen der gesprochenen Sprache auf. Es geht in der Tat eher um eine kommunikationswissenschaftliche, diskursanalytische Arbeit, die selbstverständlich linguistische Aspekte mit einbezieht; vor dem Hintergrund des Titels kommt die Linguistik allerdings zu oft zu kurz.

Bei der Beurteilung dessen, was höflich bzw. höflicher ist, spielen individuelle Auffassungen bestimmt eine Rolle. So vergleicht die Vf.in z.B. die beiden Aufforderungen Tag et stykke kage mere! [Nimm noch ein Stück Kuchen!] und Du kunne vel ikke tanke dig et stykke kage mere? [Du könntest dir wohl nicht vorstellen, noch ein Stück Kuchen zu nehmen?] $]^{3}$, wobei letztere Aufforderung weniger höflich sein sollte, weil die Aufforderung nicht deutlich genug“ (S. 41) sei. Unserer Meinung nach ist die zweite Aufforderung durchaus

2 Schwarz, Ch. (1985). Bedingungen der sprachlichen Kommunikation (= Linguistische Studien 131). Berlin: VEB Verlag Enzyklopädie.

3 Die Übersetzungen dienen lediglich der Veranschaulichung der sprachlichen Mittel im Dänischen. 
deutlich genug, was wohl auch der Grund sein dürfte, daß Fredsted sie überhaupt als solche, also als Aufforderung einstuft.

Fredsted wirft, wie bereits erwähnt, die Frage auf, ob Höflichkeit nur dann als Strategie eingesetzt wird, wenn eine Bedrohung im Sinne von face-threatening vorliegt, wie es Brown und Levinson meinen. Fredsted erscheint es plausibler, anzunehmen, daß Höflichkeit als Komponente aller Sprachhandlungen auftreten kann. Es wäre von Vorteil gewesen, wenn diese durchaus plausible Annahme (anhand von Beispielen) ausführlicher diskutiert worden wäre.

Manchmal verfolgt die Vf.in ihre eigenen Überlegungen nicht konsequent genug. Auf Seite 331 sagt sie beispielsweise, daß letzten Endes immer kontextuelle Faktoren darüber entschieden, ob eine Äußerung höflich sei oder nicht. Auf Seite 58 geht die Vf.in jedoch von der Annahme aus, daß es eine ganz geringe Anzahl von sprachlichen Formulierungen gebe, die an sich entweder höflich oder unhöflich sind.

Die Vf.in versucht u.a., die externe Kommunikation in der Tourismusbranche mit Hilfe der Fachsprachendefinition von Lothar Hoffmann einzukreisen (S. 11 ff.). Sie stellt fest, daß das gewisse Probleme bereite. Aus unserer Sicht wäre es zweckmäßiger, wenn sie Kalverkämpers Sichtweise bei dem Begriff Fachsprache herangezogen hätte (Gemeinsprachlichkeit und Fachsprachlichkeit seien keine absoluten Gegensätze, sondern sie sind vielmehr skalar aufzufassen), da sie mit seiner Definition nicht mit den von ihr angesprochenen Problemen konfrontiert gewesen wäre.

Im Literaturverzeichnis vermissen wir die Arbeiten von Sperber/Wilson, List/Wagner und, falls man die Untersuchung zur Fachsprachenforschung in Beziehung setzen möchte - was uns vernünftig zu sein scheint -, auch die von Kalverkämper. An verschiedenen Stellen diskutiert Fredsted den Begriff indirekte Sprechhandlung; hier vermißt man einen Hinweis auf Sökeland. Als Defizit muß auch die weitgehende Nichtbeachtung der funktional-pragmatischen Kommunikations- und Diskursanalyse der beiden letzten Dezennien in Deutschland (Ehlich, Redder, Rehbein u.a.) angesehen werden, die die Abhandlung wahrscheinlich nicht nur in eine dem Titel angemessenere linguistische Richtung gelenkt, sondern ihr auch nützliche Anregungen zu einer aussagekräftigeren Transkription gegeben hätte. Es soll allerdings gerechterweise betont werden, daß die transkriptionsmäßige Leistung von Fredsted sehr begrüßenswert ist, und es stellt sich zudem die Frage, ob die Grenzen des zu Erwartenden bei einer Dissertation in dieser Arbeit nicht sowieso überschritten sind.

Wir haben auf einige Probleme in Fredsteds Buch hingewiesen. Das darf aber keineswegs darüber hinwegtäuschen, daß Analyser af dansk og tysk talesprog ein gewichtiger Beitrag zur empirischen Diskursforschung ist; das Buch wird 
sicherlich Anregungen zu neuen Forschungsbeiträgen geben. Sehr wichtig scheint uns, daß die Theorien, die Fredsted ausführlich - mitunter reichlich ausführlich - referiert, anhand konkreter Texte überprüft werden.

Zuletzt nur noch die Frage, ob sich denn kein dänischer Verlag finden ließe für die von der dänischen Forschungsgemeinschaft für Geisteswissenschaften unterstützen Veröffentlichung einer dänischen, dänischsprachigen Doktorarbeit? (Gut, daß es Norwegen gibt!)

\section{Literatur}

Heinemann, Wolfgang \& Dieter Viehweger (1991). Textlinguistik. Eine Einführung (= Reihe Germanistische Linguistik. Kollegbuch. 115). Tübingen: Niemeyer.

Kalverkämper, Hartwig (1990). Gemeinsprache und Fachsprachen - Plädoyer für eine integrierende Sichtweise. In: Stickel, Gerhard (Hrsg.) (1990). Deutsche Gegenwartssprache: Tendenzen und Perspektiven (= Jahrbuch 1989. Institut für deutsche Sprache). Berlin / New York: Walter de Gruyter. 88-133.

List, Pia \& Johannes Wagner (1992). Nationale Stereotype im internationalen beruflichen Alltag: Überlegungen anhand eines Fallbeispiels. In: Grindsted, Annette \& Johannes Wagner (Hrsg.) (1992). Communication for Specific Purposes (= Kommunikation und Institution 21). Tübingen: Narr. 210-226.

Sökeland, Werner (1980). Indirektheit von Sprechhandlungen. Eine linguistische Untersuchung (= Reihe Germanistische Linguistik 26). Tübingen: Niemeyer.

Sperber, Dan \& Deidre Wilson (1995). Relevance. Communication and Cognition. Oxford / Cambridge: Blackwell. 